\title{
LEARNING GAZE DIRECTION PERCEPTION - AN INVESTIGATION BY BEHAVIORAL AND NEUROCOMPUTATIONAL APPROACHES
}

\author{
Hirokazu DOI ${ }^{1)}$, Kazuhiro UEDA ${ }^{2)}$, and Kazuyuki SHINOHARA ${ }^{1)}$ \\ ${ }^{1)}$ Nagasaki University, Japan, ${ }^{2)}$ The University of Tokyo, Japan
}

\begin{abstract}
The gaze direction perception is an important component of human social cognition, but the ontogenesis of this perceptual ability is not well known. The present study attempted to investigate the ontogenesis of the well-documented characteristics of the human gaze direction perception that the head orientation information influences the perceived gaze direction. The results showed that a simple connectionist model of the human visual system could simulate several important aspects of human performance after trained by the supervised-learning, indicating that the postnatal perceptual-learning plays some roles in the development of the gaze direction perception.
\end{abstract}

Key words: connectionist model, gaze direction, development

Directing the social attention to the proper location in the environment is indispensable for children in acquiring broad range of knowledge and skills. For example, Masataka, Koda, Urasopon and Watanabe (2009) argued the possibility that the acquisition of new skills might be accomplished in macaque populations by a rather simple mechanism of making the learners attentive to the presence or attention focus of the teacher of the skills. Also in human infants, observing the adults' gaze and emotional expression gives them opportunities to learn information about unfamiliar objects (Hoehl, Wiese, \& Striano, 2008). On the basis of these, it seems plausible to think that it is one of the major goals of learning science to clarify the mechanism of and promote the acquisition of the ability to control social attention properly.

The primary source of information regarding the social attention is the other's gaze direction, and thus it has been the topics of intensive research to investigate the perceptual mechanism underlying the gaze direction perception (Langton, 2000; Baron-Cohen, 1995; Langton, \& Bruce, 1999; Senju, Hasegawa, \& Tojo, 2005; Doi \& Ueda, 2007; Doi, Ueda, \& Shinohara, 2009; Doi, Sawada, \& Masataka, 2007). Recent research revealed several core neural regions recruited in gaze direction perception including the amygdale (Kawashima et al., 1999), the superior temporal sulcus (Calder et al., 2007), the fusiform face area (George, Driver, \& Dolan, 2001), and the intra-parietal sulcus (Haxby, Hoffman, \& Gobbini, 2000).

At the same time, there is still a significant controversy over the ontogenesis of these neural functions. Some studies favor the view that the basic functions required to perceive gaze direction are available at the neonatal stage (Batki, Baron-Cohen, Wheelwright,

Correspondence concerning this article should be addressed to Hirokazu Doi, Graduate School of Biomedical Sciences, 1-12-4 Sakamoto-cho, Nagasaki City, Nagasaki, Japan (e-mail: kazuyuki@nagasakiu.ac.jp) 
Connellan, \& Ahluwalia, 2000; Farroni, Csibra, Simion, \& Johnson, 2002). On the other hand, other researchers take a bit more empiricist view. They assert that innate implementation in the infants' brain predispose them to attend reflexively to important social signals, i.e. conspecifics' faces, in the environment, which makes the developing brain more efficient at processing the social signals such as others' gaze directions through the perceptual learning (see for a brief review, Shultz et al., 2003). One important presumption of this view is that some aspects of gaze direction perception can be acquired through the postnatal perceptual learning. Otherwise, there is no room for the postnatal interaction with the environment to play any roles in the maturation of the ability to perceive gaze direction. However, this presumption has not been tested empirically, partly because it is ethically problematic to control the visual input of gaze information to developing brain.

The present article reports our preliminary attempt to examine whether some aspects of gaze direction perception can be acquired through postnatal perceptual learning. To achieve this goal, in Experiment 1, we examined one well-documented characteristics of the human gaze direction perception, i.e. the influence of head orientation on the perceived gaze direction, by psychophysical experiment, and depicted the specific nature of this perceptual phenomenon in detail. Then, in Experiment 2, the possibility was examined that the ability to integrate the head orientation information in computing the gaze direction could be acquired through the perceptual learning by a simulation experiment using a simple connectionist model of the human visual system. If the ability could be learned through the postnatal experience, the model should be able to simulate the data in Experiment 1.

\section{EXPERIMENT 1}

The previous researches on the gaze direction perception repeatedly demonstrated that the perceived gaze direction is not necessarily determined solely by the local feature of eye region; the perceived direction of gaze is systematically affected by head orientation. In their pioneering study, Gibson and Pick (1963) showed that head rotation had a significant effect on the participants' judgment of perceived gaze direction.

William Wollaston (as cited in Langton, 2000) created a unique illusion by making use of the influence of head orientation on the perceived gaze direction. He pasted the identical eye region onto two different heads, which were directed in slightly different orientations. Of the resultant two face pictures, one was perceived to be looking straight at the viewer, while the other averted. This demonstration clearly illustrates that the identical eye regions can be perceived to be both averted and straight according to the head contexts combined with them.

The primary aim of the present experiment is to further investigate the specific nature of the Wollaston-type illusion. Of particular interest are the follwing two points. First, the recruitment of the configural mode of the face processing in the perceptual process, through which the head orientation influences the perceived gaze direction, was 


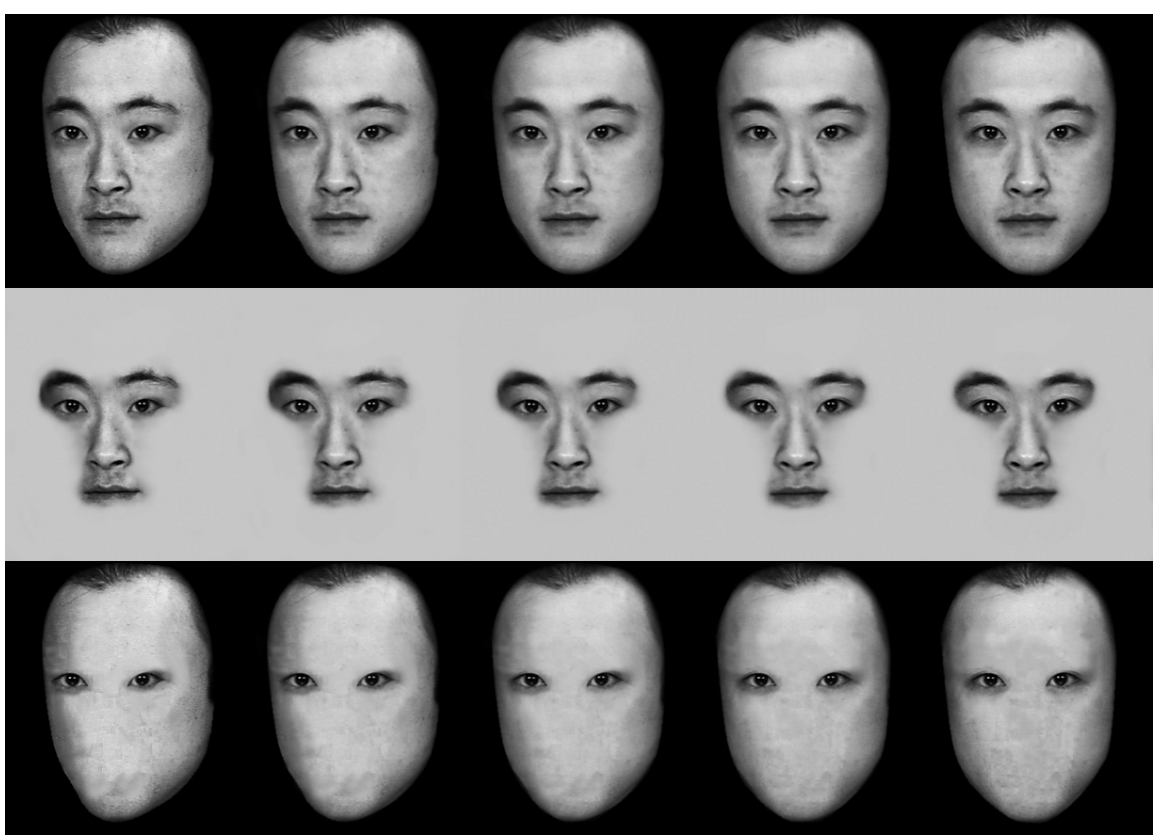

Fig. 1. The representative images of the image sequence in Normal (the upper row), Contour (the middle row), and Nose (the lower row) conditions.

investigated. The configural mode of processing is one of the characteristics of face recognition in adults (Maurer, Le Grand, \& Mondloch, 2002), and is known to be severely impaired by inverting the face. On the basis of this, we examined whether the influence of the head orientation is susceptive to the face inversion in the present experiment. The second aim was to identify the facial feature that is the most important cue in perceiving the head orientation, and thus influences the perceived gaze direction. Riccardelli and Driver (2008), classified the sources of head orientation into three categories: a) morphological information from the local eye region, b) the pointing direction of the nose, c) the head contour. In the present experiment, we erased either the b) the pointing direction of the nose, or c) the head contour from the morphed faces, which are shown in the lower and middle row of Figure 1, respectively, and measured the point where the participants judged that the gaze direction changed from "straight" to "averted". The eradication of the facial feature that exerts great influence on the perceived gaze direction should cause a discernible difference to the point at which the perceived gaze direction changed from "straight" to "averted". We crossed the face orientation and the type of information indicating the head orientation orthogonally, which makes it possible to examine whether the influence of each type information source on the perceived gaze direction is mediated by the configural mode of the face processing independently. 


\section{METHOD}

\section{Particpants}

Eight females and five males $(M=$ age 27.8 yrs old, $S D=5.6)$ participated in this study after giving informed consent for the experimental procedure, which was approved by the institutional ethical committee of Nagasaki University. Written consents were obtained from them. They were all right-handed with normal or corrected-to-normal visual acuity.

\section{Apparatus and Stimuli}

The stimuli sequences were created on the basis of Type (Full-Nose-Contour) $\times$ Orientation (UprightInverted) $\times$ Model $(2)=12$ pairs of face pictures with 0 deg and $30 \mathrm{deg}$ head contexts. The face image sequences were created on the basis of these pairs of faces. The procedure of creating the stimulus sequence is schematically depicted in Figure 2. The two pictures constituting each pair were face images with its head being directed to the straight ( 0 deg head context) and averted in $30 \mathrm{deg}$ (30 deg head context). The head context of the facial images in the Full Type condition was the normal head contexts with all the facial features clearly visible, while the nose was erased in the Nose Type head contexts. In the Contour Type head context, the contour of the head was erased and the boundary between the face and the background was blurred. The example images of each condition are shown in Figure 1. The three types of face images in each model had the identical eye region with its pupil being located at the center of the eye sockets. The location in the image on which the eye region was pasted was set exactly the same. Thus, on the 0 deg head context the gaze direction was perceived to be straight, while averted when the eye region was pasted onto the 30 deg head context. One of the two models was female and the other male. The original pictures were taken from the ATR DB99 database.

The face image sequence in each condition was created by morphing each pair of the face images by using morphing software that creates face images of $(100-x) \%$ influence of the 30 deg head context and $\mathrm{x} \%$ influence of the $0 \mathrm{deg}$ head context with a predetermined percentage of $\mathrm{x}$. The image sequences comprising of 26 face images were created by changing $\mathrm{x}$ from 0 to 100 with the step of four. The gaze direction of the face images changed from "straight" to "averted", when the image sequence was viewed in an descending order of $\mathrm{x}$. In the Upright Orientation condition, these image sequences were shown in the normal orientation, while in the Inverted condition the image sequence were presented upside-down.

Pair of Original Pictures

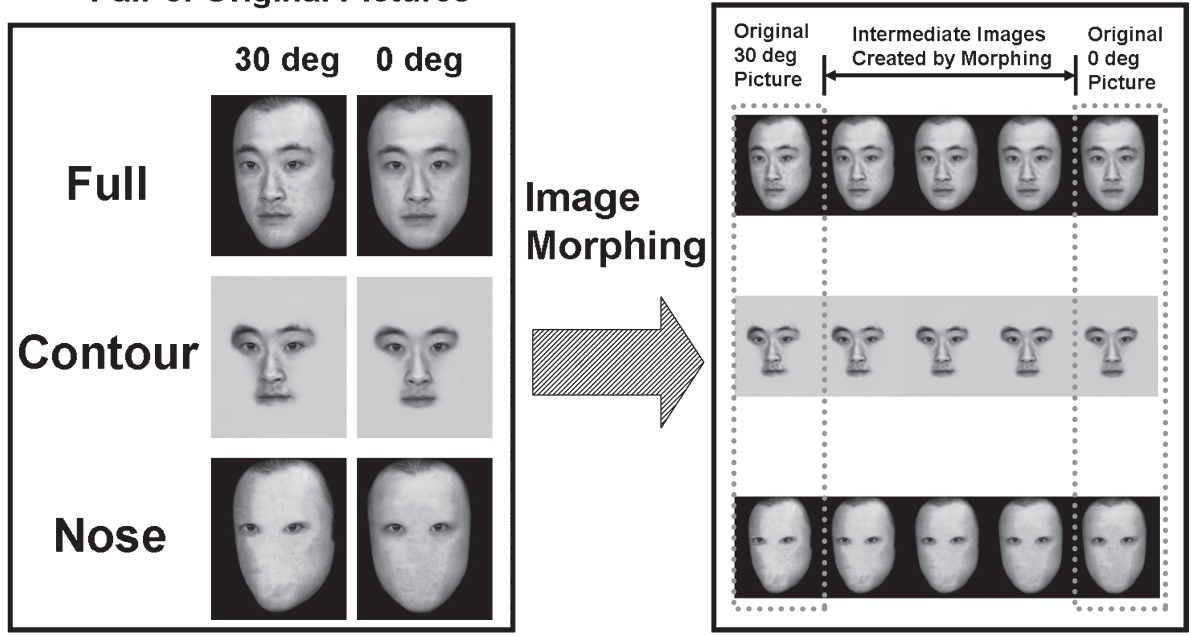

Fig. 2. The schematic depiction of the procedure of creating the stimulus sequences in each condition. 
Procedure

The stimuli are presented at the center of a 17 inch monitor on a laptop computer. Below the window where the face image was presented was a trackbar that could be moved by a mouse. At the beginning of each trial, a random dot mask was presented for $500 \mathrm{~ms}$. Then, the $\mathrm{x}=100$ face image was presented. As the trackbar was moved to the right, the head orientation of the face image changed gradually from 0 deg towards $30 \mathrm{deg}$, and vice versa; the $\mathrm{x}$ of the face image presented was changed by the trackbar. The participants were instructed to adjust the trackbar to the point where the perceived gaze direction of the face image changed from "straight" to "averted". When the participants felt confident of their adjustment, they clicked a button presented just below the trackbar and proceeded to the next trial.

The whole experiment consisted of two blocks, and there were a total of 72 experimental trials to ensure that all Orientation $(2) \times$ Type $(3)=$ six conditions were administered 12 times each. In half of the trials in each condition, the face images of the female model were presented, and in the remaining half those of the male model. The six conditions were presented pseudo-randomly. All the face images were shown in the original orientation, not in the mirror-reversed orientation.

\section{RESULTS AND DISCUSSION}

The point of judgment at which the participants judged that the perceived gaze direction changed from "straight" to "averted" in each condition is shown in Figure 3. The larger point of judgment indicates that the head orientation exerts strong influence on the gaze direction; the perceived gaze direction changes from "straight" to "averted", even when the head orientation is deviated from the straight line only slightly. These values were entered into a two-way within-participant analysis of variance (ANOVA) with the factors of Type (3) and Orientation (2). The ANOVA revealed a significant main effect of Orientation, $F(1,12)=5.46, p<.05$, with the point of judgment being larger in the Upright orientation than in the Inverted orientation. The main effect of Type also yielded significance, $F(2,24)=13.14, p<.01$. Post hoc comparisons revealed that the point of

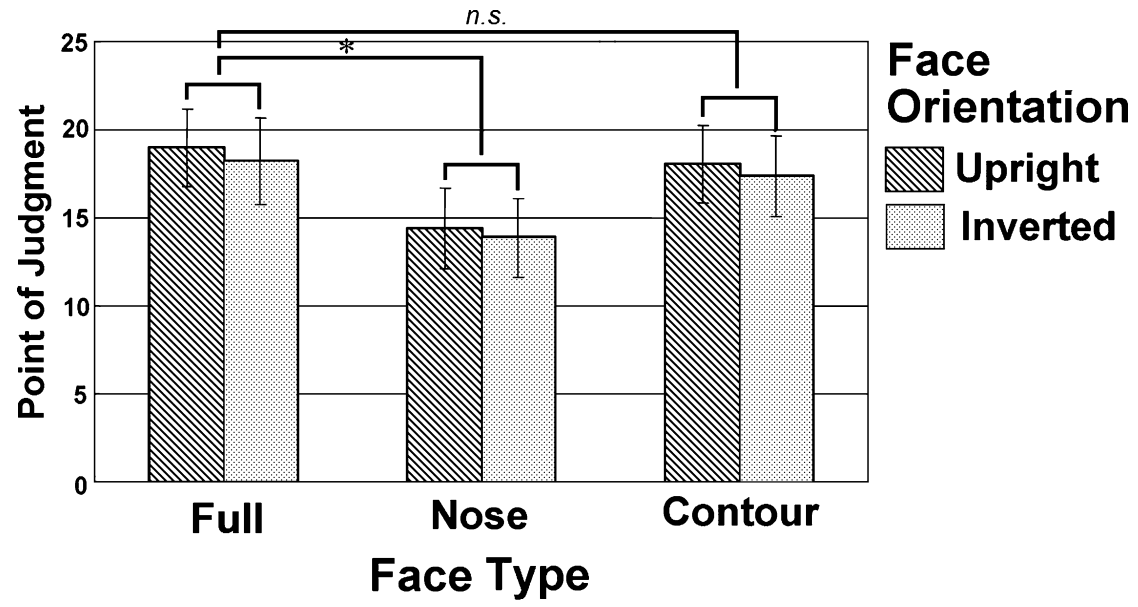

Fig. 3. The averaged point where the participants judged that the perceived gaze direction changed in each condition. The bars indicate \pm 1 SEM. $* * p<.01$. 
judgment in the Nose Type condition was significantly smaller than in the Full Type and Contour Type conditions, $p s<.05$, but no other comparisons reached significance, $p s>.10$.The interaction between Orientation and Type did not reach significance, $F(2$, $24)=0.09, p>.10$. In sum, these results indicate that the influence of the head orientation on the perceived gaze direction is indeed mediated by the configural mode of face processing. Moreover, of the two types of information examined in the present study, the nose pointing had more pronounced effect on the perceived gaze direction.

\section{EXPERIMENT 2}

The primary goal of Experiment 2 is to examine whether the data obtained in Experiment 1 could be acquired through perceptual learning process by conducting a connectionist simulation. This attempt is motivated by the recent demonstration that a simple connectionist model of the visual system comprised of a few layers simulates the seemingly high-level characteristics of the human face perception (Tong, Joyce, \& Cottrell, 2008; Dailey, Cottrell, Padgett, \& Adolphs, 2002).

In the experiment, a three-layer network model first learned to extract the gaze direction information from facial images by the back-propagation algorithm. Considering the fact that the gaze and head move independently from each other, the face database was created so that the gaze and head orientations were crossed orthogonally. After the completion of the learning, the stimulus faces presented to the human participants in Experiment 1 were fed to the connectionist model as input. The outputs of the connectionist model, the perceived gaze direction of the facial stimuli computed by the model, were compared with those of the human participants. Only the facial stimuli in upright conditions were fed to the model, because the model does not have the chances to observe the inverted faces in the training phase. If some characteristics of the gaze direction perception could be acquired through the perceptual learning, it is possible that the several features of the data obtained in Experiment 1 is simulated by the present connectionist model.

\section{METHOD}

\section{Stimulus Preparation}

Two females and four males $(M=29.3$ yrs old, $S D=4.5)$ served as the models. The acquisition of the images was undertaken in an ordinary lecture room with the settings of a white board, a digital camera, and a white canvas. Each model sat facing a white board, and the digital camera recorded the model's face. The white canvas was situated behind the model and served as the background. On the white board, the marks were presented indicating the locations $0,5,15,25,35 \mathrm{deg}$ to the right in the visual angle from the model's point of view. In the recording, we adapted the recording method suggested by Weidenbacher, Layher, Strauss, \& Neumann (2007). Specifically, we mounted a laser pointer on the model's head, and instructed the model to adjust the head orientation so that the red dot of the pointer aligns vertically with the mark representing the designated head orientation. As soon as the head orientation was adjusted, the model was instructed to fixate on the mark indicating the designated gaze direction. Following this procedure, we acquired five head orientation $\mathrm{x}$ five gaze direction $=25$ pictures for each model . 


\section{Training and Testing}

The connectionist model is a feed-forward network consisting of three-layers similar to most object recognition models (McCleery et al., 2008; Tong et al., 2008; Dailey et al., 2002). The first layer, i.e. the input layer, simulates the response properties of the neurons in the primary visual cortex (Jones \& Palmer, 1987), while the hidden layer corresponds to the later visual cortices including the fusiform face area and the superior temporal cortex. Input layer is comprised of two classes of neurons, each of which constitute the "Face Analyzer" and "Eye Analyzer" respectively. In the face analyzer, the $50 \times 50$ grayscaled pictures of the whole faces were first processed by Gabor filtering at eight different frequencies $(0, \pi / 8, \pi / 4,3 \pi / 8, \pi / 2$, $5 \pi / 8,3 \pi / 4,7 \pi / 8)$ and five different scales $(1,2,3,4,5)$. The intensity of each filtered image was sampled from 100 grids with the size of $5 \times 5$ pixels, resulting in a $100 \times 8 \times 5=4000$ dimensional representation of each image. The dimensions are compressed by the principal component analysis (PCA) into 30 principal components. Similarly, the eye analyzer processes the $125 \times 50$ grayscaled pictures of the eye regions with the same set of Gabor filters, and compresses the information into 30 principal components. The resultant $30+30=60$ principal components were fed to the hidden layer consisting of 15 nodes, which used the standard logistic sigmoid function.

The 20 networks, each with different random initial weights, were trained to output the gaze direction of the input image in degrees. The input into the network consisted of Gaze Orientation (5) $\times$ Head Orientation $(5) \times \operatorname{Model}(6)=150$ pairs of the pictures of the whole face and the corresponding eye region. The network was trained for 10000 epochs, and the error was minimized by a supervised-learning implementing the back-propagation algorithm. After the training, the networks were fed as the input the pairs of i) the stimulus picture presented in Experiment 1 and ii) the picture of the eye region cropped from them, and computed the gaze direction of each facial stimuli. Note that the stimulus pictures presented in Upright condition in Experiment 1 consisted of Type (3) $\times$ Model (2) $\times$ Influence of Head Orientation $(26)=156$ pictures. All these pictures, together with the eye regions cropped from them, served as the input to the connectionist model.

\section{RESUlTS AND DisCUSSION}

The outputs of the 20 networks to each input were averaged to yield the estimate by the network of the perceived gaze direction of each facial stimuli. In order to evaluate the effects of head orientation on the perceived gaze direction, the proportional influence of the $i$ deg head orientation was calculated by the following equation for the three (FullNose-Contour) Type conditions.

Proportional Influence

$=($ Perceived Gaze Direction in $i$ deg Head Context - Perceived Gaze in 0 deg Head Context)/Perceived Gaze in 0 deg Head Context $\times 100$

The 25 head orientations were aggregated into five ranks (from 25.2 to 30 deg head context in Rank 1; from 19.2 to 24 deg head context in Rank 2; from 13.2 to 18 deg head context in Rank 3; from 7.2 to 12 deg head context in Rank 4; from 1.2 to 6 deg head context in Rank 5), and the proportional influences of the head orientations were averaged within each rank in each Type condition. The averaged proportional influences in each rank in each condition are shown in Figure 4. The positive sign of the proportional change indicates that the perceived gaze direction deviated in the direction consistent with the head orientation. The larger the influence of the head orientation on the computed gaze direction is, the higher the absolute height of the bar in Figure 4 is. Therefore, this figure tells us that the influence of the head orientation is stronger in the Full Type condition than 


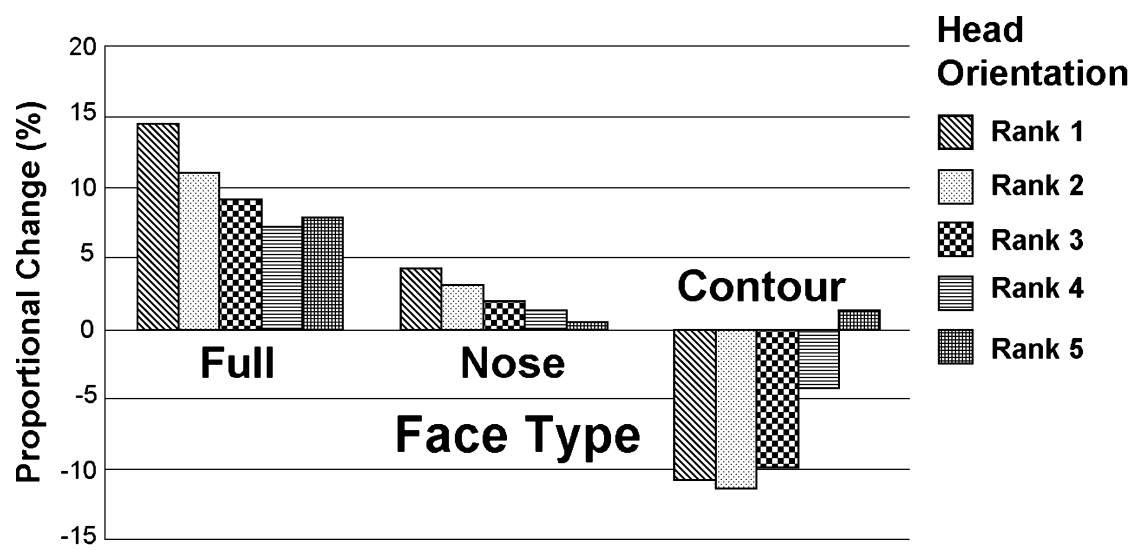

Fig. 4. The proportional influence of the head orientation in each rank in each condition.

in the Nose Type condition, which is a pattern similar to that observed in Experiment 1. Moreover, the directions of the influences in these conditions are also consistent with those observed in Experiment 1 in that the head orientation 'pulled' the perceived gaze direction. On the other hand, the direction of the influence in the Contour Type condition is apparently in the reverse direction; as the head orientation increased, the perceived gaze direction increased in the opposite direction.

\section{GENERAL DisCUSSION}

In the two preliminary experiments reported in the present article, we attempted to investigate the ontogenesis of the well-documented characteristics of the human gaze direction perception that the head orientation information influences the perceived gaze direction (Gibson, \& Pick, 1963; Langton, Honeyman, \& Tessler, 2004; Doi \& Ueda, 2007; Doi, Ueda, \& Shinohara, 2009). In Experiment 1, the results indicated that that the face inversion impairs the influence of the head context, which lends support to the contention that the influence of the head orientation on the perceived gaze direction is mediated by the configural mode of face processing (Maurer et al., 2002). Importantly, Experiment 1 further revealed that the nose pointing serves as the most distinguished cue to affect the perceived gaze direction. Langton et al. (2004) suggested that the influence of head contour is not susceptive to the face inversion. Taking these into consideration, the effect of the face orientation in the present study largely derives from the nose pointing.

In Experiment 2, the connectionist model has succeeded in simulating an important aspect of the data obtained in Experiment 1. Specifically, the influence of the head orientation was most prominent in the Full Type condition followed by the Nose Type condition. Moreover, the directions of the influences of the head orientation in these two conditions were consistent with those observed in Experiment 1. This result is as far as we 
know the first evidence to support the contention that the ability to integrate the head orientation information in computing the gaze direction could be acquired through a learning process. However, there are still some issues to be addressed before drawing any further conclusion. First, in the training phase of Experiment 2, the model learned to extract the gaze direction from the facial pictures through the supervised-learning. Then, if similar learning process takes place during the development of the human visual system, what in the environment serves as the feedback signals? To answer this question is beyond the scope of the present study, but it seems quite possible that the infants could obtain some kind of feedback signals to the accuracy of their gaze direction computation especially when they are observing not only the shift of the gaze of a viewer but the viewer's ensuing action. Consider for example the situation when a viewer looks at an object in the environment and then reaches towards it. In such situation, an infant observing this sequence of events presumably guesses the viewer's intended object from the gaze direction. If the viewer reaches towards the object that the infant first inferred to be the target of the viewer's attention, this event should serve as a kind of positive feedback signal, and vice versa. Second, the influence the head orientation exerts on the perceived gaze direction in the Contour Type condition is in opposite direction between Experiment 1 and Experiment 2. The explanation of this discrepancy is elusive at this point, but this might indicate that the influence from the head contour information is integrated into gaze direction computation with the aid of some top-down constraints. Thus, the mechanism mediating the influence of the head orientation information differs according to the type of the information that indicates the head orientation (for similar discussion, see Langton et al., 2004).

As mentioned above, the researchers have accumulated a considerable amount of data regarding the neural mechanism underlying the gaze direction perception, but there is still a significant controversy over the ontogenesis of these neural functions. The present study lends support to the contention that the postnatal perceptual-learning plays some roles in the development of the gaze direction perception by showing that a computational model of the human visual system can simulate part of the characteristics of the human gaze direction perception after a supervised-learning. Elman et al. (1996) argued in their seminal work that an innate architecture of the cortex navigates the postnatal learning, and that the development of the high-level neural functions depends on both the preprogrammed maturation of the cortex and the postnatal learning process constrained by the structure of the cortex. In the light of this framework, it seems plausible to think that in the future study the preliminary model presented in the present article can be refined to simulate the human behavior more precisely by incorporating the additional biologicallyplausible constraints into the architecture of the network.

The research on the ontogenesis of social abilities has a potential to contribute greatly to the education of mentally disabled children, because the knowledge about appropriate stimulus input, which promotes the maturation of neural mechanisms subserving social cognition, can aid practitioners to establish effective methods of early intervention for those children. Moreover, the scientific knowledge about the ontogenesis of these abilities could be of great help for researchers in judging whether a particular 
ability can be improved postnatally or not. As is correctly pointed out in Ito, Kubo-Kawai, Fukushima, Sawada, \& Masataka (2008), the investigation into "possibility of learning", i.e. whether it is possible to improve a particular ability through learning process, is indispensable in learning science; if the ability is not susceptive to educational intervention, researchers have to focus on promoting the acquisition of some alternative skills or the functional equivalence to complement the impaired ability, rather than to attempt to ameliorate the deficiency itself. Taking these into consideration, it can fairly be concluded that the burgeoning field of learning science can be further enriched by incorporating the framework that combines behavioral methodologies with neurocomputational ones as outlined in the present study.

\section{REFERENCES}

Baron-Cohen, S. 1995. Mindblindness: An essay on autism and theory of mind, MIT Press/Bradford Books, Boston.

Batki, A., Baron-Cohen, S., Wheelwright, S., Connellan, J., \& Ahluwalia, J. 2000. Is there an innate gaze module? Evidence from human neonates. Infant and Behavoral Development, 23, 223-229.

Calder, A. J., Beaver, J. D., Winston, J. S., Dolan, R. J., Jenkins, R., Eger, E., \& Henson, R. N. A. 2007. Separate coding of different gaze directions in the superior temporal sulcus and inferior temporal lobule. Current Biology, 17, 20-25.

Dailey, M. N., Cottrell, G. W., Padgett, C., \& Adolphs, R. 2002. Empath: A neural network that categorizes facial expressions. Journal of Cognitive Neuroscience, 14, 1158-1173.

Doi, H., Sawada, R., \& Masataka, N. 2007. The effects of eye and face inversion on the early stages of gaze direction perception-An ERP study. Brain Research, 1183, 83-90.

Doi, H., \& Ueda, K. 2007. Searching for a perceived stare in the crowd. Perception, 36, 773-780

Doi, H., Ueda, K., \& Shinohara, K. 2009. Neural correlates of the stare-in-the-crowd effect. Neuropsychologia, 47, 1053-1060.

Elman, J. L., Bates, E. A., Johnson, M. H., Karmiloff-Smith, A., Parisi, D., \& Plunkett. K. 1996. Rethinking innateness: A connectionist perspective on development. (Cambridge MA: MIT Press)

Farroni, T., Csibra, G., Simion, F., \& Johnson, M. H. 2002. Eye contact detection in humans from birth. Proceedings of the National Academy of Sciences of the United States of America, 99, 9602-9605.

George, N., Driver, J., \& Dolan, R. 2001. Seen gaze-direction modulates activity in fusiform face area and its coupling with other brain areas during face processing. NeuroImage, 13, 1102-1112.

Gibson, J. J., \& Pick, A. D. 1963. Perception of another person's looking behavior. The American Journal of Psychology, 76, 386-394.

Hoehl, S., Wiese, L., \& Striano, T. 2008. Young infants' neural processing of objects is affected by eye gaze direction and emotional expression. PLOS ONE, 3, e2389.

Haxby, J. V., Hoffman, E. A., \& Gobbini, M. I. 2000. The distributed human neutral system for face perception. Trends in Cognitive Sciences, 4, 223-233.

Ito, H., Kubo-Kawai, N., Fukushima, M., Sawada, R., \& Masataka, N. 2008. Learning science where cognitive neuroscience meets psychotherapy. Psychologia, 51, 280-289.

Jones, J. P., \& Palmer, L. A. 1987. An evaluation of the two-dimensional Gabor filter model of simple receptive fields in cat striate cortex. Journal of Neurophysiology, 58, 1233-1258.

Kawashima, R., Sugiura, M., Kato, T., Nakamura, A., Hatano, K., Ito, K., Fukuda, H, \& Nakamura, K. 1999. The human amygdala plays an important role in gaze monitoring: A PET study. Brain, 122, 779-783.

Langton, S. R. 2000. The mutual influence of gaze and head orientation in the analysis of social attention direction. Quarterly Journal of Experimental Psychology Series A, 53, 825-845.

Langton, S. R., \& Bruce, V. 1999. Reflexive visual orienting in response to the social attention of others. Visual Cognition, 6, 541-567.

Langton, S. R. H., Honeyman, H., \& Tessler, E. 2004. The influence of head contour and nose angle on the 
perception of eye-gaze direction. Perception \& Psychophysics, 66, 752-771.

Masataka, N., Koda, H., Urasopon, N., \& Watanabe, K. 2009. Free-ranging macaque mothers exaggerate tool-using behavior when observed by offspring. PLOS ONE, 4, e4768.

Maurer, D., Le Grand, R., \& Mondloch, C. J. 2002. The many faces of configural processing. Trends in Cognitive Sciences, 6, 255-260.

McCleery, J. P., Zhang, L., Ge, L., Wang, Z., Christiansen, E. M., Lee, K., \& Cottrell, G. W. 2008. The roles of visual expertise and visual input in the face inversion effect: Behavioral and neurocomputational evidence. Vision Research, 48, 703-715.

Ricciardelli, P., \& Driver, J. 2008. Effects of head orientation on gaze perception: how positive congruency effects can be reversed. The Quarterly Journal of Experimental Psychology, 61, 491-504.

Senju A, Hasegawa T, \& Tojo Y. 2005. Does perceived direct gaze boost detection in adults and children with and without autism? The stare-in-the-crowd effect revisited" Visual Cognition, 12, 1474-1496.

Schultz, R. T., Grelotti, D. J., Klin, A., Kleinman, J., Van Der Gaag, C., Marois, R., \& Skudlarski, P. 2003. The role of the fusiform face area in social cognition: Implications for the pathobiology of autism. Philosophical Transactions of the Royal Society B: Biological Sciences, 358, 415-427.

Tong, M. H., Joyce, C. A., \& Cottrell, G. W. 2008. Why is the fusiform face area recruited for novel categories of expertise? A neurocomputational investigation. Brain Research, 1202, 14-24.

Weidenbacher, U., Layher, G., Strauss, P., \& Neumann, H. 2007. A Comprehensive Head Pose And Gaze Databas. In 3rd IET International Conference on Intelligent Environments, Ulm (Germany), September 2007. 